\title{
A Comparative study of the etiology of symptomatic vaginal discharge based on WHO syndromic diagnosis and microbiological diagnosis
}

\author{
Nisha Bhatia ${ }^{1, *}$, Krishna Kumari Meka ${ }^{2}$, Manick Dass .S. ${ }^{3}$, DVLK Swetha ${ }^{4}$ \\ ${ }^{1}$ Assistant Professor, ${ }^{2,3}$ Professor and HOD, ${ }^{4}$ Student, ${ }^{1,2}$ Dept. of Obstetrics and Gynecology, ${ }^{3,4}$ Dept. of Microbiology, Apollo \\ Institute of Medical Sciences and Research, Hyderabad, Telangana, India
}

*Corresponding Author:

Email: nish_178@yahoo.co.in

\begin{abstract}
Introduction: Vaginal discharge is the most common complaint with which women of reproductive age group present to the gynecological outpatient. WHO syndromic approach algorithm helps in identifying the etiology based on the gross appearance of discharge but it often fails to detect the etiology correctly. Addition of simple microbiological tests helps to diagnose the etiology accurately.

Objective: To compare the diagnosis of symptomatic vaginal discharge based on WHO syndromic algorithm and microbiological tests.

Materials and Methods: 100 Women in the age group of 18 to 45 years with symptomatic vaginal discharge attending the gynecological OPD of Apollo Institute of Medical Science and Research, Hyderabad were examined in the outpatient and a clinical diagnosis made using WHO syndromic algorithm for vaginal discharge. Under aseptic precautions, vaginal discharge was collected and subjected to simple lab tests such as Gram's stain and direct microscopy (wet mount). The lab results were compared with the clinical diagnosis.

Results: Among 100 women, bacterial vaginoses was the most common diagnosis. In 55\% of patients etiological agent could be identified by lab investigations. By WHO syndromic approach, bacterial vaginosis was over diagnosed (70 vs. 44 cases confirmed by lab) and candidiasis was slightly under diagnosed (8 vs. 10 cases by lab). The sensitivity of WHO syndromic approach in diagnosing bacterial vaginosis is $100 \%$ while specificity is only $53 \%$. The difference in diagnosis rate of candidiasis clinical versus microbiological diagnosis was marginal (10\% vs. 11\%). Trichomoniasis was accurately diagnosed by WHO syndromic approach.

Conclusions: WHO syndromic algorithm for vaginal discharge over diagnoses bacterial vaginosis but has a high sensitivity and specificity in diagnosing candidiasis and trichomoniasis. Simple microbiological test such as Gram stain and wetmount will increase the accuracy in diagnosis thereby preventing overtreatment.
\end{abstract}

Keywords: Vaginal discharge, WHO syndromic approach, Microbiological tests.

\section{Introduction}

The most common complaint of a woman in reproductive age group presenting to Gynecology outpatient is excessive white discharge. It has a prevalence of around $30 \% .^{1}$ Vaginal discharge could be either physiological or pathological. The most common causes of symptomatic vaginal discharge are bacterial vaginosis followed by candidiasis and trichomoniasis. ${ }^{2}$ Pathological discharge can lead to significant morbidity such as pelvic inflammatory disease, infertility and preterm labour. Hence an accurate diagnosis is very essential. Simple lab tests such as Gram's stain of the vaginal smear for bacterial vaginosis and candidiasis and direct microscopy (wet smear) of the vaginal discharge for trichomonasvaginalis have been found to be very useful in diagnosing the etiological agent and focusing on specific treatment. ${ }^{3}$

\section{Aim}

The present study was conducted to compare the diagnosis based on WHO syndromic algorithm ${ }^{4}$ and microbiological tests in the etiology of symptomatic vaginal discharge.
Study Design: Cross sectional study

Sample: 100 Women in the age group of 18 to 45 years with symptomatic vaginal discharge attending the gynecological OPD of Apollo Institute of Medical Science and Research, Hyderabad were enrolled in this study.

Inclusion Criteria: All sexually active women between 18 to 45 years of age with symptomatic vaginal discharge willing for a vaginal examination.

Exclusion Criteria: Women using intrauterine contraceptive device or using oral contraceptive pills. Women with co morbidities like urinary tract infection or diabetes, previous gynecological surgeries

\section{Materials and Methods}

Cusco's vaginal speculum, sterile bottles, sterile cotton swabs, sterile glass slides and microscope. Patients presenting with excessive vaginal discharge attending the Gynecology OPD of Apollo Institute of medical science and Research (AIMSR General Hospital) were enrolled in this study. They were examined by speculum examination and the discharge was diagnosed based on WHO syndromic approach which classifies vaginal discharge based on colour and consistency. The etiology was classified based on the 
gross appearance of the discharge- bacterial vaginosis profuse watery grayish white discharge, candidiasisthick curdy white discharge; Trichomoniasis- Profuse frothy greenish yellow and Indeterminate if they had mixed features. The discharge from the vagina was collected using the vaginal speculum and tested for bacterial vaginosis, candidiasis and trichomoniasis by standard simple microbiological procedures bacterial vaginosis diagnosed by Gram's stain score of seven or more based on the Nugent scoring system. Candidiasis: if gram positive budding yeasts and pseudohyphae were seen on gram's stain. trichomoniasis: if wet smear microscopy was positive for motile trichomonas vaginalis.

\section{Results}

This prospective study included 100 patients with symptomatic vaginal discharge who were examined in gynecology OPD at AIMSR for a period of one year (2016). Bacterial vaginosis was common in age group of 20-30 while candidiasis remained most prevalent between the age groups of 30-40. Age did not have any statistically significant association with vaginal discharge.

70 patients were diagnosed as bacterial vaginosis based on profuse greyish white discharge. Similarly 8 samples were diagnosed as candidiasis due to thick curdy white discharge and 1 sample was clinically diagnosed as trichomoniasis based on the profuse yellowish appearance of discharge and strawberry spots on cervix.

Table 1: WHO syndromic diagnosis and etiology of vaginal discharge

\begin{tabular}{|l|c|}
\hline \multicolumn{1}{|c|}{ Condition } & $\begin{array}{c}\text { WHO Syndromic } \\
\text { Diagnosis }\end{array}$ \\
\hline Bacterial vaginosis & 70 \\
\hline Candidiasis & 8 \\
\hline Trichomoniasis & 1 \\
\hline Indeterminate/Mixed & 21 \\
\hline
\end{tabular}

In $55 \%$ of patients diagnosis could be made by microbiological examination when compared to $45 \%$ of patients where no significant microbiological findings, pointing at a particular pathogen were found.

Table 2: Microbiological diagnosis and etiology of vaginal discharge

\begin{tabular}{|l|c|}
\hline \multicolumn{1}{|c|}{ Condition } & $\begin{array}{c}\text { Microbiological } \\
\text { diagnosis }\end{array}$ \\
\hline Bacterial Vaginosis & 44 \\
\hline Candidiasis & 10 \\
\hline Trichomoniasis & 1 \\
\hline Intermediate flora & 15 \\
\hline Cannot be determined & 30 \\
\hline
\end{tabular}

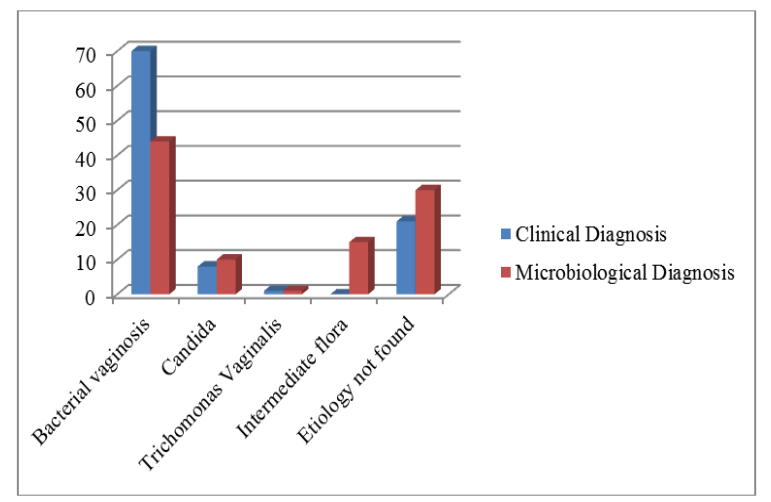

Fig. 1: WHO syndromic diagnosis and microbiological diagnosis of symptomatic white discharge

Out of the 100 patients, clinically 70 of them were diagnosed to have bacterial vaginosis, whereas microbiological findings showed 44 out of them to actually have bacterial vaginosis. Similarly 8 patients were clinically diagnosed with candidiasis whereas 10 of them showed positive microbiological findings. On the other hand trichomoniasis showed high correlation with the clinical and microbiological findings. This was found to be statistically significant. (p-value<0.05) by chisquare test.

Bacterial vaginosis diagnosis of bacterial vaginosis based on clinical features was correlated with microbiological diagnosis gold standard-Nugent score.

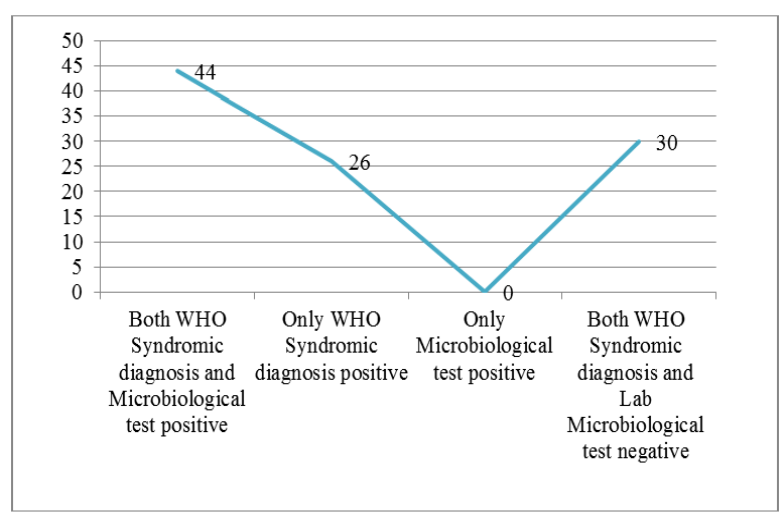

Fig. 2: WHO syndromic approach and microbiological test in diagnosing bacterial vaginosis

The sensitivity of WHO syndromic approach in diagnosing bacterial vaginosis is $100 \%$ while specificity is only $53 \%$. 


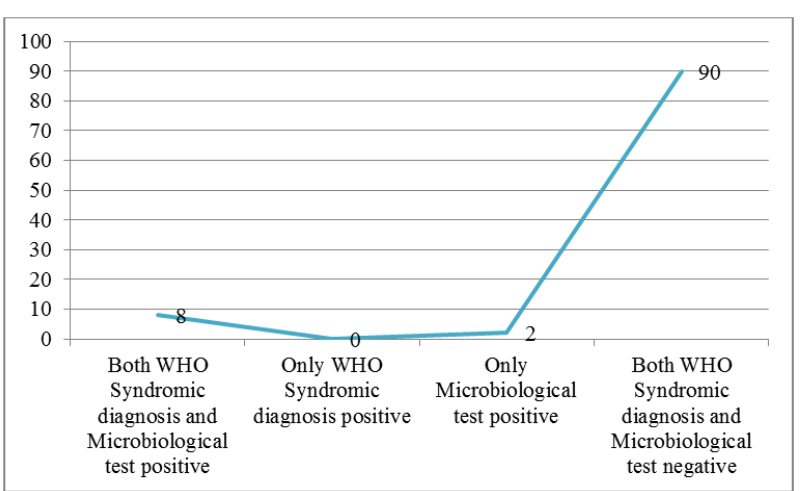

Fig. 3: WHO Syndromic approach and microbiological test in diagnosing candidiasis

WHO syndromic approach had sensitivity of 83\% in identifying Candidiasis and a specificity of $100 \%$.

Trichomoniasis- WHO syndromic approach clinically diagnosed one case of trichomoniasis which was confirmed by wet mount on microbiology.

\section{Discussion}

In the present study, we studied 100 women of reproductive age group who presented to Gynecological OPD with excessive vaginal discharge. The most common etiology in our study was bacterial vaginosis followed by candidiasis and trichomoniasis. This observation was similar to most of the studies conducted worldwide. ${ }^{3,4}$

WHO syndromic approach in identifying etiology of vaginal discharge was used in the present study. Bacterial vaginosis was over diagnosed (70 vs. 44 cases confirmed by lab) and candidiasis was slightly under diagnosed ( 8 vs. 10 cases by lab). A review by Pettifor et $\mathrm{al}^{5}$ in 2000 states that the algorithmic approach to diagnosis has a good performance with sensitivity ranging from 73 to $93 \%$ in patients with Symptomatic vaginal discharge. We found similar results in our study too. The causative agent was identified in $55 \%$ of the patients, in the remaining $45 \%$ patients; microbiological diagnosis could not be made. Ray et $\mathrm{al}^{6}$ also stated that in patients with symptomatic vaginal discharge only $37.5 \%$ had a confirmed etiological diagnosis. This was similar to studies conducted by Ryan, C. $\mathrm{A}^{7}$ and Nugent, R.P ${ }^{8}$ which showed that in $12-54 \%$ of the patients complaining of vaginal discharge, diagnosis could not be reached using any of the diagnostic approaches.

The most common clinical diagnosis based on WHO syndromic approach was bacterial vaginosis in our study, which was about $70 \%$ followed by mixed infections, Candida infection and trichomoniasis. In 21 cases the etiology of discharge couldn't be diagnosed clinically. This was similar to a study done by Karaca et $\mathrm{al}^{9}$ that also showed $29 \%$ mixed clinical infections clinically.

Bacterial vaginosis was clinically diagnosed by presence of homogenous discharge clinically. In our study the diagnosis could be confirmed microbiologically in 44 cases only while in the rest 26 it could not be confirmed since clue cells were not seen. In study done by Karaca et $\mathrm{al}^{9} \quad 27.8 \%$ confirmed microbiologically to have bacterial vaginosis as compared to $66 \%$ which remain undiagnosed. In an Indian study done in 2014 by Rekha et al $^{10}$ bacterial vaginosis was clinically diagnosed in $47 \%$ of cases while microbiologically confirmed only in $24 \%$ of cases, which was almost similar to our study.

Candida could be correctly diagnosed clinically due to the typical curdy white discharge in 8 cases while microbiology could pick up 10 cases. This observation was also similar to study by Rekha et al ${ }^{10}$ where the difference in diagnosis rate of clinical versus microbiological diagnosis was marginal (10\% vs. $11 \%)$

Trichomoniasis in our study was diagnosed clinically in 1 patient due to presence of profuse yellowish discharge with itching and strawberry spots on cervix and it was confirmed by microbiology thereby sensitivity and specificity was very high similar to study by Landers et al $^{11}$ which revealed high sensitivity and specificity to clinical diagnosis as compared to microbiological diagnosis as gold standard. One third of cases of trichomoniasis are asymptomatic which could have been a limitation of this study as we included only patients with symptomatic vaginal discharge. Moreover, although wet mount examination is inexpensive and rapid, its sensitivity is low, when compared to culture. ${ }^{12}$

Hence our study revealed that if clinical diagnosis is considered as a sole test to diagnose etiology of vaginal discharge we would be over diagnosing bacterial vaginosis in patients since the WHO syndromic approach has high sensitivity but low specificity. Ray K et $\mathrm{al}^{6}$ also reported high sensitivity of the syndromic approach for vaginal discharge syndrome, but the specificity of this method in diagnosing vaginal discharge was low. This means that we would be misdiagnosing cases of physiological white discharge for bacterial vaginosis and over treating them by unnecessary antibiotics. ${ }^{13}$ For trichomoniasis and candidiasis, clinical diagnosis is reliable as it has high sensitivity and specificity. This observation was in contrast to studies done in other centers which show that clinical diagnosis has moderate sensitivity for candidiasis and lower sensitivity for trichomoniasis.

\section{Conclusion}

Treatment based on WHO syndromic approach over diagnoses cases as bacterial vaginosis but has a high sensitivity and specificity in diagnosing candidiasis and trichomoniasis. Combining clinical diagnosis with simple lab tests will reveal the exact etiology and help in differentiating physiological from pathological causes thereby aiding in specific targeted treatment of symptomatic vaginal discharge. 


\section{References}

1. Centers for Disease Control and Prevention. Sexually transmitted diseases treatment guidelines 2006.

2. Thulkar J, Kriplani A, Aggarwal N, et al. Indian J Med Res. 2010;131:83-87.

3. Choudhry S, Ramachandran VG, Das S, Bhattacharya SN, Mogha NS. Pattern of sexually transmitted infections and performance of syndromic management against etiological diagnosis in patients attending the sexually transmitted infection clinic of a tertiary care hospital. Indian J Sex Transm Dis. 2010;31:104-8.

4. Geneva, Switzerland: WHO; 2003. World Health Organization. Guidelines for the Management of Sexually Transmitted Infections; pp. 1-60.

5. Pettifor A, Walsh J, Wilkins V, et al. How effective is syndromic management of STDs?: A review of current studies. Sex Transm Dis 2000;27:371-385.

6. Ray K, Muralidhar S, Bala M, Kumari M, Salhan S, Gupta SM, et al. Comparative study of syndromic and etiological diagnosis of reproductive tract infections/sexually transmitted infections in women in Delhi. Int J Infect Dis. 2009;13(6):e352-9.

7. Ryan CA, Zidouh A, Manhart LE, et al. Sex Transm Infect. 1998;74:95-105.

8. Nugent RP, Krohn MA, Hillier SL. Reliability of diagnosing bacterial vaginosis is improved by a standardized method of gram stain interpretation. J Clin Microbiol 1991;29:297-301.

9. Karaca M, Bayram A, Kocoglu ME, Gocmen A, Eksi F. Comparison of clinical diagnosis and microbiological test results invaginal infections. Clin Exp Obstet Gynecol. 2005;32(3):172-4.

10. Rekha S, Jyothi, et al. Comparison of visual, clinical and microbiological diagnosis of symptomatic vaginal discharge in the reproductive age group. Int J Pharm Biomed Res. 2010;1:144-148.

11. Landers DV, Wiesenfeld HC, Heine RP, et al. Predictive value of the clinical diagnosis of lower genital tract infection in women. Am J Obstet Gynecol 2004;190:1004.

12. Patil, M.J.,Nagamoti JM, Metgud SC. Diagnosis of trichomoniasis vaginalis from Vaginal Specimens by Wet mount Microscopy, in Pouch TV culture system, and PCR, J Glob Infect Dis. 2012 Jan;4(1):22-5.

13. Patel V, Pednekar S, Weiss H, Rodrigues M, Barros P, Nayak B, et al. Why do women complain of vaginal discharge? A population survey of infectious and pyschosocial risk factors in a South Asian community. Int J Epidemiol. 2005;34:853-62. 